\title{
Analysis and Correction of the abnormality of the pitch angle's residual error in the Ka system
}

\author{
Zheng Liu , Huihui Zou , Fuan Su \\ China Satellite Tracking and Controlling Department, Jiangyin 214431, China \\ liuzheng354@qq.com
}

Keywords: Ka; residual error; pitch angle

Abstract. Based on the problem of abnormality of the pitch angle's residual error in the dynamic calibration process of the Ka system, this article analyzes reasons of the problem from many aspects. It checks reasons one by one and finally determines the main cause of the problem. Through the parameter correction method, it restores mechanism of the problem, which has important referential significance to a similar situation in follow-up equipment.

\section{Introduction}

The measurement accuracy of the ship measurement and control equipment is not only related to the servo ability. It is also related to factors, including the deformation of the equipment, ships' yaw and the target's dynamic characteristics. Meanwhile, it is difficult to accurately separate error factors. Therefore, before the official use of the shipboard measurement and control equipment by new research and transformation, it usually needs to do its accuracy identification and dynamic tracking performance verification through static calibration and dynamic calibration. The measurement system should be calibrated with calibration data to identify error factors in the calibration process. Through the data analysis and processing, the shaft error model, dynamic error model and ship error model can be obtained. In the measurement process, data correction results can be improved, improving the accuracy of target measurement in official use. In the process of dynamic calibration, there is abnormality of the pitch angle's residual error in the Ka system. The residual curve of odd voyage shows the opposite trend with the even voyage. To find out the root cause of the problem, the elevation data will be modeled and analyzed.

\section{Pitch error sources}

The error sources of the shipborne measurement and control equipment are complex. There are many influencing factors, which not only include the common errors of model error, equipment error and tracking error of the target, but also the errors by ship shaking, hull deformation and ship position. The definition of residuals in the national standard is the sum of random errors and uncorrected systematic errors. Therefore, the standard calibration residuals mean the difference between the high-precision GPS data and the corrected measurement data. Its focus is on the accuracy of GPS data and measurement data. In order to determine the accuracy of GPS, by analysis of the tracking data of different devices on the same voyage, it is found that the pitch residual curves of other devices are symmetrical and there is no abnormal phenomenon. Similarly, to identify the accuracy of the measured data before correction, the Ka system's saving data is analyzed and drawn. The pitch angle's error curve is symmetrical and there is no abnormality. Therefore, this abnormality may come from the error correction.

\section{Establishment of the pitch error model}

Based on the position and principles of pitch error, with the combination of data processing methods and processes, the theory and method of data statistics are used. Some error correction models are established in advance to do hypothesis tests on calibration results and distribution rules of error 
coefficients of equipment. At the same time, we continue to use calibration results to update these models to make improvement.

The correction principle of the pitch error model. The error correction model of pitch measurement data is a mathematical expression to describe the variation law of the coefficient of the correctable coefficient. Its key point lies in getting model structures and coefficients of each systematic error. For different equipment and systems, different correction models should be established. For the Ka system, examples are shown as in the following figure.

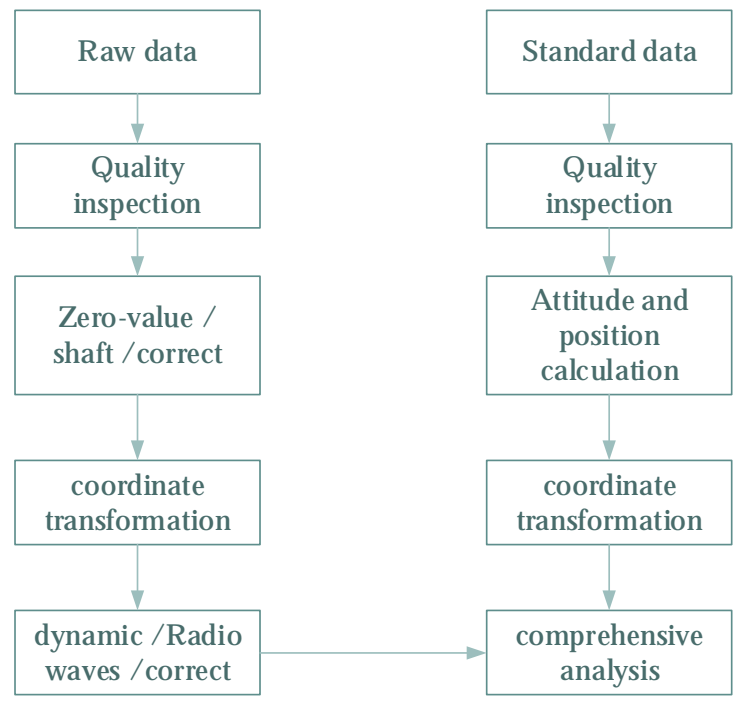

Fig. 1 Flow chart of error correction

Parameter identification of the error model. Zero error is usually because that the initial reading is not standard and other factors. After the zero angle calibration, when the electric axis points to the north direction, the azimuth and pitch's reading is the zero angle error. This error depends on the calibration devices and calibration. It is also related to the measurement accuracy of the fiducial mark and the triaxial center. The zero value error makes that the angle's indication value is inconsistent with the target's true value, which needs to be corrected by the model parameter. The zero value error is generally used as the determination constant.

$$
\left\{\begin{array}{l}
R=R_{\text {实测 }}-\Delta R_{0} \\
E=E_{\text {实测 }}-\Delta E_{0} \\
A=A_{\text {实测 }}-\Delta A_{0}
\end{array}\right.
$$

The base surface is not horizontal or uneven sinking, which can lead to the deformation of the antenna base. Vibration and climate can lead to the deformation of the antenna base. If the in-horizontal degree is $\theta_{\mathrm{m}}$, the degree of the pitch and the horizontal plane is also $\theta_{\mathrm{m}}$. The azimuth of the antenna during rotation is $A$. The large surface's largest non-horizontal position Angle is $A_{n}$. By the spherical trigonometry function, the angle error caused by the large surface's non-horizontal angle $\theta_{\mathrm{m}}$ is as follows:

$$
\Delta E=\theta_{\mathrm{m}} \bullet \cos \left(A \mathrm{~A}_{\mathrm{m}}\right)
$$

The rotation axis's stiffness of the antenna is poor rigidity. There is severe dynamic deformation. Due to wind and high-speed rotation, there is much shaking. It makes that the azimuth axis and the pitch axis of the antenna are not orthogonal. If the non-orthogonality is $\delta$, by the spherical trigonometry function, the azimuth error caused by the non-orthogonal degree $\delta$ is as follows.

$$
\Delta E=\delta \bullet \tan E
$$


The calibration of the electric axis depends on the optical axis. The photoelectric deviation can naturally lead to the inaccuracy of the measurement data. The photoelectric deviation is divided into the horizontal angle component $K_{\mathrm{z}}$ and the longitudinal angle component $K_{\mathrm{e}}$. The pitch angle error which is caused by the vertical component $K_{\mathrm{e}}$ of the photoelectric deviation is $K_{\mathrm{e}}$ itself.

The gravitational sag is caused by the change of the relative position of each component of the antenna structure due to gravity. It mainly makes the antenna's gain decrease and it makes the antenna's minor lobe increase. At the same time, there is the electric axis shift. The gravitational sag error affects only the pitch angle error. If the gravitational sag error is $E_{\mathrm{mg}}$, its pitch angle error is as follows.

$$
\Delta E=E_{\mathrm{mg}} \bullet \cos \left(E_{\mathrm{c}}+E_{0}\right)
$$

When the radio wave is transmitting in the atmosphere, due to the different media in the atmosphere, the different refractive indices can bend the electromagnetic wave propagation path. It can makes the electric axis points to the direction of the different target. The difference can be calculated based on the formula. The empirical formula is as follows.

$$
\Delta E_{N}=N_{\mathrm{s}}^{0} \cot E_{\mathrm{c}}(\mathrm{rad})
$$

In the dynamic measurement process of shipborne measurement and control equipment, due to the influence of ship shaking and the ship position, the measurement data can be mixed with abnormal signals. It is usually periodical and it has an impact on measurement factors R, A, E. In the test process, the use of high-precision GPS data to determine the ship position and the servo system to meet the target requirements of isolation of the ship shaking, the error can be ignored.

In tracking the fast target, due to the limitations of the servo system bandwidth, the axis can not be timely and ready to track the target. And the target is always deviating from the axis of an angle, which results in a dynamic hysteresis error. The classic correction formula is as follows.

$$
\Delta \theta=\frac{\Delta U}{\mu}
$$

Through the above analysis of the error model parameter, it can be known that all parameters are real-time dynamic. To simplify the system model, usually the linear treatment should be done. By the theoretical derivation, the ship measurement system's pitch angle error model is as follows.

$$
E=E_{c}+E_{0}+\theta_{m} \cos \left(A_{c}+A_{0}-A_{m}\right)+K_{e}+E_{m g} \cos \left(E_{c}+E_{0}\right)+k \bullet \frac{\Delta U_{e}}{\mu_{e}}-\Delta E_{N}
$$

The azimuth elevation is not orthogonal, which mainly affects the azimuth error. In the dynamic lag, the error voltage value's absolute value tends to be zero. The zero error and optoelectronic deviations affect the size of the pitch angle system. However, it will not lead to abnormality in the pitch residual trend. Therefore, main reasons of abnormalities include in-horizontal large surface, gravitational sag and wave re-fractions.

\section{Influence of error parameters on residual abnormalities}

Through the comparison and analysis of standard calibration data, we establish the appropriate relationship model between the error and influence. We statistically test the model structure and reliability to test the compactness, dynamics and smoothness of the model. The influence weight of each error parameter is determined. It can provide the data support for further processing of the elevation residual error.

Zero-value error on the impact of residual anomalies. There are two different values used for the zero value of the Ka system's equipment. The zero value error can affect the system error of pitch angle. It does not lead to the trend of abnormalities in the pitch residual. Comparing the two parts of 
the pitch residual value separately, we can know that the zero-value error is not a major factor in the anomalous tendency of pitch residuals.

Radio waves' impacts on residual anomalies. The normal flight distance of the Ka system is generally within the appropriate range designed in advance. The AGC voltage usually varies with the distance. By observing the AGC curve of two voyage with abnormal trends, it is found that there is no significant correlation between the trend of AGC voltage and the trend of pitch residual error. Therefore, it can be concluded that the radio waves' transmitting error is not the main factor of the abnormal trend of the pitch residual error.

Analysis of the impact of the shaft erron on the model. Shaft parameters mainly include photoelectric deviation, gravitational sag and in-horizontal large surface. In the same tracking mode, two kinds of electro-optical deviation pitch residual curves are respectively analyzed. It is found that they are abnormal. However, other parameters such as gravitational sag and non-horizontal large surface are collected through dynamic calibration in real time. Among them, the stability of gravitational sag parameter is good. And the maximum tilt amount and maximum tilt azimuth of the large surface do not have great changes. Through comparison of the initial data, we cannot judge the impact of the large surface's horizontal level on the pitch residual error, which needs further analysis and researches.

\section{Test verification}

After the above data processing and analysis, to further prove that the abnormal trend of pitch residual error is caused by the in-horizontal large surface, the data of different voyage tracks are not corrected. Because the trend of the slip of the sixth voyage curve is obvious, its amount of the in-horizontal level's change is easy to be inferred. Through correcting the maximum tilt of the in-horizontal large surface, you can basically eliminate the trend of curve tilt. Abnormal data in other voyage is processed in the same way, which can also basically eliminate the tendency of curve tilt.

\section{Conclusions}

By analyzing the mechanism of pitch angle error, with the combination of identification of model parameters, a dynamic model of pitch error correction is established. After it is determined that the in-horizontal large surface has a great influence on the pitch residual anomaly in shaft parameteres, the in-horizontal level is corrected. The abnormal status can be basically eliminated. The flight process is dynamic and the internal and external environment is complex. The modified model is limited and the dynamic performance is not enough. Therefore, the corrected system residual still takes a certain amount of weight. The statistical error method needs to be applied to do further calibration. Meanwhile, it needs to further refine the correction model.

\section{References}

[1] Yang Lei. Error Separation and Analysis of Instrumentation Ship-Measured Data [J]. Journal Of Spacecraft TT \& C Technology, 2008, 27(1):30-34.

[2] Guo Deqiang. Analysis and treatment of residual data from space measurement ship[D]. Master Degree Thesis of National University of Defense Technology, 2008 .

[3] Tu Xianqin. The Statistics of Measurement Noise of the Instrumentation Ship[J]. Journal Of Spacecraft TT \& C Technology, 2006, 25(3):72-77.

[4] Li Xiaoyong. Error Separation for Dynamic Measurement Data of Ship-borne Radiometric Equipment I [J]. Journal Of Spacecraft TT \& C Technology, 2009, 28(2):71-77. 